\title{
Atomistic Simulations of Surface Effects Under High Electric Fields
}

\author{
Stefan Parviainen ${ }^{1}$, Michal Dagan ${ }^{2}$, Shyam Katnagallu ${ }^{3}$, Baptiste Gault ${ }^{3}$, Michael Moody ${ }^{2}$, François
} Vurpillot ${ }^{1}$

${ }^{1 .}$ GPM UMR 6634 CNRS, Université et INSA de Rouen, Rouen, FRANCE

2. Department of Materials, University of Oxford, UK

${ }^{3 .}$ Max-Planck Institut für Eisenforschung, Düsseldorf, Germany

The dynamics of a surface changes drastically when it is exposed to an intense electric field. In the case of a metal, an external electric field will induce a charge on the surface, leading to interaction between the field and the surface. This can lead to the deformation of the surface due to tensile stress from the interaction, but also more subtle effects, such as lowered barriers for diffusion of surface ad-atoms, field assisted evaporation of atoms from the surface and significant changes in the local electric field.

These effects are very important in several applications. For example, Field Ion Microscopy (FIM) and Atom Probe Tomography (APT) are atomic-resolution microscopy techniques that rely on the controlled evaporation of atoms from a surface by an external electric field, to determine the shape (and in APT also the composition) of a studied sample. In the case of APT atoms from the sample itself are pulled from the surface by the electric field, while in the case of FIM an imaging gas is used instead. In both cases the evaporated atoms are ionized and accelerated toward a detector by the electric field. The shape of the sample can then be deduced with near-atomic resolution by assuming a simple shape for the trajectories[1].

However, in reality, the trajectories of the evaporated atoms are not so simple, and may be distorted by the local environment. In APT the sample shape is constantly evolving as atoms are evaporating from the sample itself, resulting in a significant change in the local electric field over time. In FIM this is not an issue, but even in this case the surface shape does not remain constant - as even in this case migrating atoms can change the surface geometry. Thus some of the simple assumptions used in data analysis may not hold.

As it is currently not possible to study the shape of the local electric field on the atomic level experimentally, we instead rely on computational methods. To be able to study the kind of effects described above, we have extended a Molecular Dynamics (MD) code to include the effect of an external electric field on a metallic surface. This is achieved by dynamically calculating the induced charge on surface atoms, corresponding to a given applied external electric field. The force resulting from the interaction between the electric field and the charged atom is then added to the classical interatomic interactions included in classical molecular dynamics. Thus, it is possible to use the technique to simulate any metallic sample for which a suitable MD interatomic potential exists.

In this work we present two sets of results, described below to illustrate the applicability of the simulation technique. In both cases we compare our computational results with experiments. In the first case we show how the surface ad-atom migration of $\mathrm{W}$ on $\mathrm{W}$ is modified in the presence of an electric field. In the second case we show how the removal of one or more surface atoms alters the local electric field, making it difficult to interpret experimental FIM images. 
Our results show that in the presence of an external electric field, ad-atom migration can be enhanced by almost an order of magnitude on a W sample. The explanation for this is that the electric field pulls the migrating atom away from the surface, weakening the interaction with the other surface atoms, and allowing it to move more freely on the surface. The calculated surface diffusion coefficient increases with increasing field. However, once the field is sufficiently strong, it will trigger field evaporation instead of migration.

We also show that the diffusion coefficient increases with time, as the migrating atoms drift towards edges, where the local electric field is enhanced due to geometric effects. The presence of an ad-atom will further increase the field enhancement near a ledge, raising the probability of atoms from a lower plane climbing up the ledge, or triggering the evaporation of the ad-atom and nearby atoms.

Another effect we describe is the local distortion in the electric field between field evaporation events. As atoms evaporate one by one from a sample, the local electric field changes. Experimentally it is observed in FIM images that when an atom is evaporated, remaining nearby atoms seem to move significantly on the surface. This was initially explained as the atoms relaxing into new equilibrium positions. However, our simulations show that the relaxation of atoms is not enough to explain the observed motion alone. Instead, as the local atomic environment changes, the change in the direction of the local electric field is sufficiently large to alter the trajectories of the subsequently evaporated ions. Thus, the experimentally observed "motion" seems to be the result of an aberration in ion trajectories as opposed to actual movement on the surface. The conclusion is that much care must be taken when interpreting FIM images of changing surfaces.

In addition to the above described cases, the simulation code is flexible enough to be used to study a wide variety of situations. Future work will include studying the effect of field induced strain on field evaporation, the deformation of surfaces in the presence of near-surface voids and precipitates, as well as fracture of samples under very high fields, which is a common problem in APT.

\section{References:}

[1] P. Bas et al, Appl Surf. Sci. 87/88 (1995), p. 298.

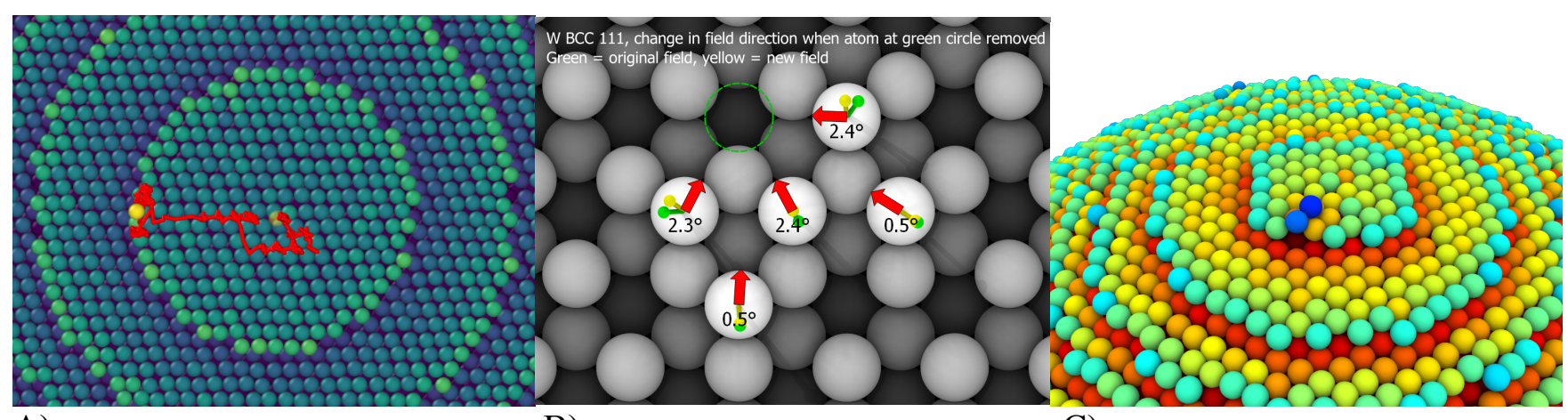

A)

B)

C)

Figure 1. A) Simulated migration path of a $\mathrm{W}$ atom on a $\mathrm{W}$ sample under a high electric field. Due to geometric field enhancement the field is higher at the edge, leading to a directed migration. B) Calculated change in the local electric field when one atom is removed from a W (111) surface. The change in the direction is several degrees, which affects the trajectories of any evaporating atoms. C) A migrating $\mathrm{W}$ atom enables another $\mathrm{W}$ atom to climb up a ledge an a simulated $\mathrm{W}$ sample. 